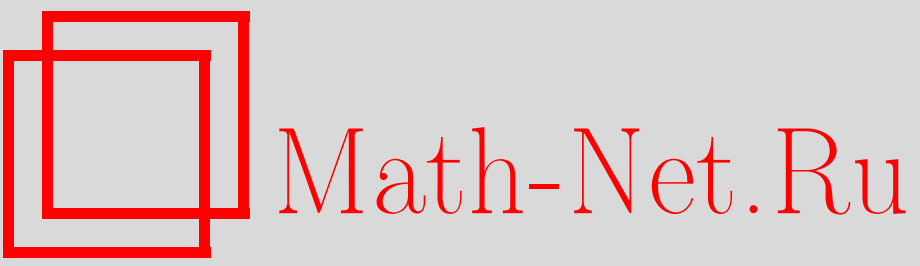

И. Ю. Попов, Е. С. Тесовская, Электрон в магнитной структуре: асимптотика резонанса, ТМФ, 2006, том 146, номер 3, 429-442

DOI: https://doi.org/10.4213/tmf2045

Использование Общероссийского математического портала Math-Net.Ru подразумевает, что вы прочитали и согласны с пользовательским соглашением

http://www . mathnet.ru/rus/agreement

Параметры загрузки:

IP : 3.85 .5 .30

26 апреля 2023 г., 16:49:26 
ТЕОРЕТИЧЕСКАЯ

И МАТЕМАТИЧЕСКАЯ

ФИЗИКА

Том 146, № 3

март, 2006

(c) 2006 г.

И. Ю. Попов*, Е. С. Тесовская*

\section{ЭЛЕКТРОН В МНОГОСЛОЙНОЙ МАГНИТНОЙ СТРУКТУРЕ: АСИМПТОТИКА РЕЗОНАНСА}

Рассмотрен оператор Лапласа с граничным условием Неймана для двумерных слабо связанных полос. Получена асимптотика квазисвязанного состояния, близкого к границе ветви непрерывного спектра. В рамках асимптотического подхода изучена задача рассеяния. Обсуждаются возможные приложения результатов.

Ключевые слова: волновод, спектр, рассеяние, резонанс.

\section{1. ВВЕДЕНИЕ}

Проблема связанных состояний и резонансов для слабо связанных квантовых проводов широко обсуждается. При анализе поведения баллистических электронов в соответствующих полупроводниковых наноструктурах она сводится к спектральной задаче для оператора Гельмгольца (оператора Шредингера свободной частицы) в сложной области с граничными условиями Дирихле. Некоторые результаты здесь уже получены. А именно, вариационные оценки для связанных состояний (не для резонансов), близких к нижней границе непрерывного спектра, найдены в работах [1], [2]. Асимптотика связанных состояний для случая условий Дирихле получена в работах [3], [4]. Вариационные оценки связанных состояний для комбинированных условий Дирихле-Неймана есть в статье [5]. Что касается резонансов (квазисвязанных состояний), соответствующие асимптотики для случая Дирихле построены в работах [6], [7]. Некоторые оценки для резонансов (случай Дирихле) найдены в работе [8]. Естественный вопрос, касающийся аналогичной проблемы для других краевых условий, оставался открытым. Это не абстрактный математический вопрос, поскольку он связан с физическими задачами. Действительно, если мы имеем дело только с полупроводниковыми структурами, условие Дирихле (обнуление волновой функции электрона на границе) является наиболее естественным. В случае же металлических наноструктур, таких как магнитные и немагнитные слои, ситуация меняется.

${ }^{*}$ Санкт-Петербургский государственный университет информационных технологий, механики и оптики, Санкт-Петербург, Россия. E-mail: popov@mail.ifmo.ru, kate-ts@mail.ru 
Недавние экспериментальные исследования многослойных материалов, образованных магнитными (Fe, Co, Ni) и немагнитными слоями [9], показывают, что существуют ферромагнитное и антиферромагнитное упорядочения соседних магнитных слоев, зависящие от ширины разделяющего немагнитного слоя. Это связано с осцилляциями соответствующего взаимодействия между слоями [10]. В большинстве поликристаллических образцов период осцилляций составляет около $10 \mathrm{~A}$, а амплитуда взаимодействия обратно пропорциональна квадрату ширины немагнитного слоя. Данное взаимодействие обычно анализируют в рамках модели квантовых ям. Эти ямы образованы магнитными слоями, которые ограничивают движение электронов проводимости с соответствующими проекциями спина в немагнитных слоях. Существование таких ям показано экспериментально для слоев из $\mathrm{Cu}$ на поверхности $\mathrm{Co}(100)$ и $\mathrm{Ag}$ на $\mathrm{Fe}(100)$ с помощью фотоэмиссии.

Рассмотрим электрон в немагнитном слое, заключенном между магнитными. Пусть состояния в магнитном слое с соответствующими направлениями спина заняты. Тогда электрон с данным направлением спина в немагнитном слое находится в потенциальной яме (в поперечном направлении). Если соседние магнитные слои намагничены одинаково, то количество ям в сверхрешетке совпадает с количеством немагнитных слоев. Ширина $L_{0}$ ямы есть ширина немагнитного слоя. Расстояние $L_{1}$ между слоями - это ширина магнитного слоя. В случае антиферромагнитного упорядочения в сверхрешетке размер ямы увеличивается и становится равным $2 L_{0}+L_{1}$. Но в этом случае яма есть для двух электронов с противоположными спинами.

Оба случая могут рассматриваться как задача о прохождении волн в волноводах с различными граничными условиями. А именно, ферромагнитное упорядочение ведет к задаче о волноводе шириной $L_{0}$ с граничными условиями Дирихле. Антиферромагнитное упорядочение ведет к задаче о двух волноводах шириной $L_{0}$ с граничным условием Неймана на разделяющей линии (мы предполагаем, что $L_{0} \gg L_{1}$, и заменяем в модели магнитный слой линией) и условием Дирихле на других границах. В настоящей статье мы рассматриваем резонансные эффекты, вызванные наличием малых отверстий в линии, разделяющей волноводы. Точнее, мы получаем асимптотическое разложение по $a$ (2a- ширина отверстия) квазисвязанного состояния (резонанса), близкого к границе $N$-й ветви непрерывного спектра для двух двумерных волноводов с граничным условием Неймана. Заметим, что при условии Неймана в отличие от условия Дирихле нет связанных состояний, есть только резонансы. Сначала мы доказываем существование резонансов, о которых идет речь. Здесь используется нестационарный подход, основанный на идеях, предложенных в работе [11]. Затем мы строим асимптотическое разложение резонанса, а также рассматриваем задачу рассеяния в рамках асимптотического подхода и описываем резонансные эффекты. Обсуждаются возможные приложения результатов в наноэлектронике. Следующие разделы посвящены обоснованию асимптотического разложения. Мы находим оценку невязки, а затем остаточного члена в разложении, используя метод, предложенный в работе [12]. 


\section{2. ТЕОРЕМА СУЩЕСТВОВАНИЯ ДЛЯ РЕЗОНАНСОВ}

Здесь мы обсудим проблему существования резонансов в случае одного отверстия связи. Система двух плоских волноводов $\Omega^{+}, \Omega^{-}$шириной $d_{+}, d_{-}$, связанных через отверстие диаметром $2 a$, рассматривается как предел при $D_{\infty}$ последовательности расширяющихся областей $D_{n}=D_{\infty} \backslash\left\{\left(x_{1}, x_{2}\right) \in \Omega^{-},\left|x_{1}\right|>n\right\}$ (при $\left.n \rightarrow \infty\right)$. Пусть $\Omega^{n}$ - соответствующая область: $\Omega^{n}=\left\{\left(x_{1}, x_{2}\right) \in \Omega^{-},\left|x_{1}\right| \leqslant n\right\}$ (резонатор шириной $d_{-}$и длиной $\left.9 n\right) ; \quad D_{n} \subset D_{n+1}$. Для такой системы задача о резонансах изучена в работах [11], [13], и получены оценки их вещественных и мнимых частей.

Пусть $L^{(0)}$ - оператор Лапласа с краевым условием Неймана для области $D_{0}=$ $\Omega^{+} \cup \Omega^{-}, \quad D_{n, 0}=\Omega^{+} \cup \Omega^{n}$ и $L^{(n)}-$ соответствующий оператор для области $D_{n}$. Рассмотрим гильбертовы пространства $L_{2}\left(D_{n}, d x_{1} \times d x_{2}\right)$. Пусть $S\left(L^{(n)}, L^{(0)}, I\right)-$ оператор рассеяния. Решение $u\left( \pm, m, \lambda, x_{1}, x_{2}\right)$ задачи рассеяния для оператора $L^{(n)}$ определяется обычным образом с использованием соответствующих функций вместо плоских волн [11]:

$$
e\left( \pm, m, \lambda, x_{4}, x_{2}\right)=\exp \left( \pm i x_{1} \sqrt{\lambda-\frac{\pi^{2} m^{2}}{d_{+}^{2}}}\right) \sqrt{\frac{2}{d_{+}}} \cos \left(\frac{\pi m x_{2}}{d_{+}}\right) \Theta\left(\lambda-\frac{\pi^{2} m^{2}}{d_{+}^{2}}\right)
$$

где $\Theta(\lambda)=1$ для $\lambda>0$ и $\Theta(\lambda)=0$ для $\lambda<0$.

ЛЕмма 1. Оператор $G_{0}(t)=e^{-t L^{(0)}}$ есть ограниченный интегралъный оператор со следующим ядром:

$$
\begin{aligned}
G_{0}\left(x_{1} \times x_{1}, x_{1}^{\prime} \times x_{2}^{\prime}, t\right)=( & \left.\frac{1}{\sqrt{2 \pi t}}\right) e^{-\left(x_{1}-x_{1}^{\prime}\right)^{2} /(4 t)} \sum_{j}\left(e^{-\nu_{j}^{+} t} \frac{5}{d_{+}} \cos \left(\frac{\pi j x_{2}}{d_{+}}\right)\right) \times \\
& \left.\times \cos \left(\frac{\pi j x_{2}^{\prime}}{d_{+} \chi_{\Omega_{+}}}\right)+e^{-\nu_{j}^{-} t} \cos \left(\frac{\pi j x_{2}}{d_{-}}\right) \cos \left(\frac{\pi j x_{2}^{\prime}}{d_{-}}\right) \chi_{\Omega_{-}}\right),
\end{aligned}
$$

где $\chi_{\Omega_{+}}, \chi_{\Omega_{-}}-$характеристическая функиия области $\Omega_{+}, \Omega_{-}$.

Пусть $\lambda_{\infty}, \varepsilon-$ числа такие, что

$$
0<\operatorname{Re} \lambda_{\infty}<\pi^{2} d_{-}^{-2}, \quad\left|\lambda-\lambda_{\infty}\right|<\varepsilon, \quad\left\{\lambda=\left|\lambda-\lambda_{\infty}\right|<\varepsilon\right\} \cap\left\{\pi^{2} j^{2} d_{-}^{-2}\right\}=\varnothing .
$$

Ниже мы обсудим выбор $\lambda_{\infty}$ более детально.

Лемма 2. Если $0<\operatorname{Im} \lambda<\pi / t$, mo оператор

$$
R_{0}(\lambda)=\left(e^{-t L^{(0)}}-e^{-t \lambda}\right)^{-4}
$$

может бить представлен в следующем виде:

$$
R_{0}(\lambda)=-e^{t \lambda}\left(I+\sum_{\nu_{j}^{ \pm}<\operatorname{Re} \lambda_{\infty}}\left(K\left(\lambda-\nu_{j}^{+}\right) P_{j}^{+} \chi_{\Omega_{+}}+K\left(\lambda-\nu_{j}^{-}\right) P_{j}^{-} \chi_{\Omega_{-}}\right)+A(\lambda)\right)
$$


где $P_{j}^{ \pm}$- интегралъный оператор в $L_{2}\left(3, d_{ \pm} ; d x\right)$ с ядром

$$
\frac{2}{d_{+}} \cos \left(\frac{\pi j x_{2}}{d_{ \pm}}\right) \cos \left(\frac{\pi j x_{2}^{\prime}}{d_{ \pm}}\right)
$$

$K(\lambda)$ - интегралъный оператор в $L_{2}\left(\mathbf{R}^{\mathbf{1}} ; \mathbf{d x}\right)$ с ядром

$$
K\left(x_{1}, x_{1}^{\prime}, \lambda\right)=\frac{i}{2 t \sqrt{\lambda}} e^{i\left|x_{1}-x_{1}^{\prime}\right| \sqrt{\lambda}}, \quad 0<\arg \sqrt{\lambda}<\pi,
$$

$A(\lambda)$ - интегралъный оператор в $L_{2}\left(D_{0} ; d x_{1} d x_{2}\right)$ с ядром, которое аналитично в круге $\left|\lambda-\lambda_{\infty}\right|<\varepsilon$ и имеет следуюшую оценку:

$$
\|A(\lambda)\|_{L_{2}\left(0, d_{+} ; 0, d_{-} ; d x_{2}\right)}<\text { const } \cdot e^{-\delta\left|x_{1}-x_{1}^{\prime}\right|} .
$$

Чтобы доказать равенство (2), мы используем разложение Фурье (по функциям $\left.\sqrt{2 / d_{+}} \cos \left(\pi j x_{2} / d_{ \pm}\right)\right)$и преобразование Фурье по $x_{1}$. Чтобы сделать обратное преобразование Фурье, сдвигаем контур интегрирования в комплексную плоскость, учитываем вычеты и оценку интеграла на контуре.

Оператор $G(t)=e^{-t L}-$ интегральный оператор в $L_{2}\left(D_{n}, d x_{1} \times d x_{2}\right)$ с ядром $G\left(x_{1}, x_{2}, x_{1}^{\prime}, x_{2}^{\prime}, t\right)$, которое удовлетворяет следующим условиям:

$$
\begin{aligned}
\frac{\partial}{\partial t} G\left(x_{1}, x_{2}, x_{1}^{\prime}, x_{2}^{\prime}, t\right) & =-L_{x_{1} x_{2}} G\left(x_{1}, x_{2}, x_{1}^{\prime}, x_{2}^{\prime}, t\right), \\
G\left(x_{1}, x_{2}, x_{1}^{\prime}, x_{2}^{\prime},+0\right) & =\delta\left(\left(x_{1}, x_{2}\right)-\left(x_{1}^{\prime}, x_{2}^{\prime}\right)\right), \\
\frac{\partial}{\partial n} G\left(x_{1}, x_{2}, x_{1}^{\prime}, x_{2}^{\prime}, t\right) & =0,\left(x_{1}, x_{2}\right) \in \partial D .
\end{aligned}
$$

Доопределим $G_{n}(t)$ нулем на $D_{0} \backslash D_{n}$. Равенство (4) будет выполнено только в области $D_{n}$. По принципу максимума получаем неравенство

$$
0 \leqslant G\left(x_{1}, x_{2}, x_{1}^{\prime}, x_{2}^{\prime}, t\right) \leqslant \widetilde{G}\left(x_{1}, x_{2}, x_{1}^{\prime}, x_{2}^{\prime}, t\right)
$$

где $\widetilde{G}$ - фундаментальное решение (4) во всем пространстве.

Пусть $V$ - соответствующий оператор $V=e^{-t L^{(0)}}-e^{-t L}\left(\right.$ или $V=G_{0}-G$ ).

ЛЕмма 3. Имеют место следующие оценки:

$$
\begin{aligned}
0 \leqslant G\left(x \times z, x^{\prime} \times z^{\prime}, t\right) & \leqslant \text { const } \cdot e^{-\delta\left|x_{1}-x_{1}^{\prime}\right|^{2}}, \\
& \left|V\left(x \times z, x^{\prime} \times z^{\prime}, t\right)\right| \leqslant \operatorname{const} \cdot e^{-\delta\left(x_{1}^{2}+x_{1}^{\prime 2}\right)}, \quad \delta>0 .
\end{aligned}
$$

Пусть $H_{ \pm}-$гильбертовы пространства с нормами

$$
\|f\|_{H_{ \pm}}^{2}=\int_{D_{0}}\left|f\left(x_{1}, x_{2}\right)\right|^{2} e^{\mp\left|x_{1}\right|} d x_{1} d x_{2} .
$$

Следующие две леммы доказываются способом, аналогичным доказательству соответствующих утверждений в работе [11]. 
Лемма 4. Оператор $V$ - самосопряженный оператор с конечным следом в $L_{2}\left(D_{0}, d x_{1} \times d x_{2}\right)$. Если рассматривать его как оператор из $H_{+}$в $H_{-}$, то это компактный оператор.

Пусть $\Gamma(\lambda)\left(\Gamma_{n}(\lambda)\right)$ есть $\Gamma(\lambda)=R_{0}(\lambda) V \quad\left(\Gamma_{n}(\lambda)=R_{0}(\lambda) V_{n}\right), \quad 0<\operatorname{Im} \lambda<\pi / t$.

Лемма 5. Оператор-функиия $\lambda \rightarrow \Gamma(\lambda) \in L\left(H_{+} \rightarrow H_{-}\right)$имеет аналитическое продолжение из области $\left\{\lambda:\left|\lambda-\lambda_{\infty}\right|<\varepsilon, \operatorname{Im} \lambda>0\right\}$ в круг $\left\{\lambda:\left|\lambda-\lambda_{\infty}\right|<\varepsilon\right\}$, и ее значения есть компактные операторы. В круге $\left\{\lambda:\left|\lambda-\lambda_{\infty}\right|<\varepsilon\right\}$ имеют место следующие соотношения:

$$
\left(G_{0}(t)-e^{-t \lambda}\right) \Gamma(\lambda)=G_{0}(t)-G(t)
$$

Приходим к следующей лемме.

ЛЕмма 6. Последовательность операторов $\left\{\Gamma_{n}(\lambda)\right\}$ сходится $\kappa \Gamma_{\infty}(\lambda)$ однородно относительно $\lambda$.

Доказательство есть простое следствие экспоненциальных оценок (см. выше):

$$
\begin{aligned}
\| \Gamma_{n}(\lambda)- & \Gamma_{\infty}(\lambda) \|_{L\left(H_{+} \rightarrow H_{-}\right)} \leqslant \mathrm{const} \cdot \int_{D_{0}}\left(G_{n}\left(x_{1}, x_{2}, x_{1}^{\prime}, x_{2}^{\prime}, t\right)-\right. \\
& \left.-G_{\infty}\left(x_{1}, x_{2}, x_{1}^{\prime}, x_{2}^{\prime}, t\right)\right) d x_{1} d x_{2} d x_{1}^{\prime} d x_{2}^{\prime} \leqslant C e^{-n \delta} \rightarrow 0 .
\end{aligned}
$$

В результате получаем следующую теорему.

Теорема 1. Оператор Лапласа с граничным условием Неймана для двумерных полос $\Omega_{ \pm}$, связанных через малое отверстие, имеет резонанс (квазисобственное значение), близкий $к$ нижней гранище ветви непрерьвного спектра $\pi^{2} d_{-}^{-2}$.

Для доказательства заметим, что резонанс есть полюс оператор-функции $\lambda \rightarrow$ $(I-\Gamma(\lambda))^{-1}$ (см., например, [11]). Задача о резонансах для области $D_{n}$ изучена в работах [11], [13]. Показано, что существуют $\lambda_{n}, \operatorname{Im} \lambda_{n}<0,0<\operatorname{Re} \lambda_{n}<\pi^{2} d_{-}^{-2}$ (резонансы), такие, что уравнение $\Gamma_{n}\left(\lambda_{n}\right) \psi_{n}=\psi_{n}$ имеет нетривиальное решение. Получены оценки для $\operatorname{Im} \lambda_{n}, \operatorname{Re} \lambda_{n}$ [13]. Чтобы доказать нашу теорему, нам не требуется полной информации об этих оценках, требуется лишь их простое следствие, а именно: существует ограниченная область (круг) на комплексной плоскости, отделенный от точки $\pi^{2} d_{-}^{-2}$, который содержит все $\lambda_{n} \quad\left(0<\operatorname{Re} \lambda_{n}<\pi^{2} d_{-}^{-2}-\epsilon\right.$, $\epsilon>0)$. Значит, в круге существует их точка сгущения. Возьмем ее в качестве $\lambda_{\infty}$ (см. выше). Существует сходящаяся подпоследовательность $\left\{\lambda_{n_{k}}\right\}, \quad \lambda_{n_{k}} \rightarrow \lambda_{\infty}$. Последовательность операторов $\left\{\Gamma_{n}(\lambda)\right\}$ сходится к $\Gamma_{\infty}(\lambda)$ (лемма 6$)$, следовательно, соответствующая подпоследовательность операторов $\Gamma_{n_{k}}$ сходится: $\Gamma_{n_{k}} \rightarrow \Gamma_{\infty}$. Поэтому $\lambda_{\infty}$ есть полюс оператор-функции $\lambda \rightarrow\left(I-\Gamma_{\infty}(\lambda)\right)^{-1}$, т.е. резонанс. 


\section{3. РЕЗОНАНСЫ: ФОРМАЛЬНОЕ АСИМПТОТИЧЕСКОЕ РАЗЛОЖЕНИЕ}

Рассмотрим систему двух плоских волноводов $\Omega^{+}, \Omega^{-}$шириной $d_{+}, d_{-}$, связанных через малые отверстия размером $2 a$. Сначала проанализируем случай двух различных волноводов (когда $d_{+}>d_{-}$), т.е. мы рассматриваем уравнение Гельмгольца с краевым условием Неймана:

$$
\Delta u+k^{2} u=0,\left.\quad \frac{\partial u}{\partial n}\right|_{\partial \Omega}=0 .
$$

ЗАмечАниЕ 1. Мы предполагаем, что решение удовлетворяет условию Неймана на всех границах, а не только на разделяющей линии. Это не существенно; важно, чтобы условие Неймана было на той линии, в которой имеется отверстие.

Нижняя граница непрерывного спектра для оператора Лапласа с условием Неймана - нуль (для лапласиана с условием Дирихле она больше нуля). Наличие отверстия приводит к появлению квазисобственного значения (резонанса) около границы второй (третьей, четвертой и т.д.) ветви непрерывного спектра. Мы используем метод согласования асимптотических разложений решений [13], [14], модификация которого для возмущения непрерывного спектра была разработана в работах [3]. Будем искать главные члены асимптотического разложения квазисобственного значения, близкого к границе второй ветви непрерывного спектра. Точнее, мы стараемся определить коэффициенты $\tau_{1}$ и $\tau_{2}$ следующего асимптотического разложения:

$$
\sqrt{\frac{\pi^{2}}{d_{+}^{2}}-k_{a}^{2}}=\tau_{1} \ln ^{-1}\left(\frac{a}{d_{-}}\right)+\tau_{2} \ln ^{-2}\left(\frac{a}{d_{-}}\right)+\cdots,
$$

где $k_{a}^{2}$ - квазисобственное значение, близкое к границе $\pi^{2} / d_{+}^{2}$, а точки означают члены более высокого порядка относительно $\ln ^{-2}\left(a / d_{-}\right)$.

Мы используем хорошо известное разложение функции Грина $G^{ \pm}(x, y, k)$ с граничным условием Неймана. Нам необходимо знать асимптотику функции Грина в окрестности особенности для значений спектрального параметра, близких к границе непрерывного спектра (для $d_{+}>d_{-}$):

$$
\begin{gathered}
G^{+}\left(x, 0, k_{a}\right)=\frac{\cos \left(\pi x_{2} / d_{+}\right)}{d_{+} \sqrt{\pi^{2} d_{+}^{-2}-k_{a}^{2}}} e^{-\left|x_{1}\right| \sqrt{\pi^{2} d_{+}^{-2}-k_{a}^{2}}}-\frac{1}{\pi} \ln |x|+g^{+}\left(x, k_{a}\right), \\
G^{-}\left(x, 0, k_{a}\right)=-\frac{1}{\pi} \ln |x|+g^{-}\left(x, k_{a}\right),
\end{gathered}
$$

$x=\left(x_{1}, x_{2}\right), x_{1}, x_{2}-$ локальные декартовы координаты (с началом в центре отверстия) точки $x, g^{+}(x, k)$ и $g^{-}(x, k)$ - функции без особенностей. Асимптотическое разложение квазисобственной функции $\psi_{a}(x)$ таково:

$$
\psi_{a}(x)= \begin{cases}\sqrt{\pi^{2} d_{+}^{-2}-k_{a}^{2}} a_{0} G^{+}\left(x, 0, k_{a}\right)+\cdots, & x \in \Omega^{+} \backslash S_{\sqrt{a}}, \\ v_{0}(x / a)+v_{1}(x / a) \ln ^{-1}\left(a / d_{-}\right)+\cdots, & x \in S_{2 \sqrt{a}}, \\ -\sqrt{\pi^{2} d_{+}^{-2}-k_{a}^{2}} a_{0} G^{-}\left(x, 0, k_{a}\right)+\cdots, & x \in \Omega^{-} \backslash S_{\sqrt{a}},\end{cases}
$$


где $S_{t}$ - круг радиуса $t$ с центром в центре отверстия, $a_{0}$ - некоторый коэффициент.

Проведем замену переменных: $\xi=x / a, \rho=|\xi|$. Процедура согласования сводится к выбору функций $v_{i}(\xi)$ таких, что $v_{i}$ - решения уравнения Лапласа с граничным условием Неймана, и члены соответствующих порядков в асимптотическом разложении совпадают. Учитывая соотношения $(7)$, получаем для $\psi_{a}^{ \pm}(x)$ (т.е. $\psi_{a}(x)$ в $\left.\Omega^{ \pm} \backslash S_{\sqrt{a}}\right)$ в окрестности отверстия

$$
\begin{aligned}
& \psi_{a}^{+}(x)=a_{0} {\left[\frac{1}{d_{+}}-\frac{\tau_{1}}{\pi}+\ln ^{-1}\left(\frac{a}{d_{-}}\right)\left(-\frac{\tau_{1}}{\pi} \ln \rho+\tau_{1} g^{+}\left(0, \frac{\pi}{d_{+}}\right)-\frac{\tau_{2}}{\pi}\right)\right]+} \\
&+ o\left(\ln ^{-1}\left(\frac{a}{d_{-}}\right)\right), \\
& \psi_{a}^{-}(x)=-a_{0}\left[-\frac{\tau_{1}}{\pi}+\ln ^{-1}\left(\frac{a}{d_{-}}\right)\left(-\frac{\tau_{1}}{\pi} \ln \rho+\tau_{1} g^{-}\left(0, \frac{\pi}{d_{+}}\right)-\frac{\tau_{2}}{\pi}\right)\right]+ \\
&+o\left(\ln ^{-1}\left(\frac{a}{d_{-}}\right)\right) .
\end{aligned}
$$

Сравнивая члены порядка $a^{0}$, видим, что подходящий выбор функции $v_{0}(\xi)$ - это постоянная $v_{0}(\xi)=a_{0} /\left(2 d_{+}\right)$. Действительно,

$$
a_{0}\left(\frac{1}{d_{+}}-\frac{\tau_{1}}{\pi}\right)=v_{0}(\xi)=a_{0} \frac{\tau_{1}}{\pi} .
$$

Следовательно, получаем

$$
\tau_{1}=\frac{\pi}{2 d_{+}}
$$

Чтобы найти $\tau_{2}$, следует рассмотреть члены порядка $\ln ^{-1}\left(a / d_{-}\right)$. Тут нам требуется функция $v_{1}(\xi)$, являющаяся решением уравнения Лапласа с краевым условием Неймана, которая имеет соответствующую асимптотику при $\xi \rightarrow \infty$. Из теории потенциала известно [15], что такая функция существует. Она может быть описана с использованием функции $X_{0}(\zeta)=\ln \left(\zeta+\sqrt{\zeta^{2}-1}\right)$ комплексной переменной $\zeta=\zeta_{1}+i \zeta_{2}$. Она гармонична, удовлетворяет условию Неймана и имеет соответствующую асимптотику: $\ln \left(\zeta+\sqrt{\zeta^{2}-1}\right)=\ln |\zeta|+\ln 2+o(1), \quad \zeta \rightarrow \infty$. Вот почему мы выбираем следующий вид функции $v_{1}(\xi)$ :

$$
v_{1}(\xi)= \begin{cases}-\frac{a_{0} \tau_{1}}{\pi} X_{0}(\xi)+c_{0}, & \xi_{2}>0, \\ \frac{a_{0} \tau_{1}}{\pi} X_{0}(\xi)+c_{0}, & \xi_{2}<0,\end{cases}
$$

где константа $c_{0}$ выбрана таким образом, чтобы согласовать соответствующие члены как в $\Omega^{+}$, так и в $\Omega^{-}$. А именно, должны иметь место следующие равенства:

$$
\left\{\begin{array}{l}
-\frac{a_{0} \tau_{1}}{\pi} \ln \rho-\frac{a_{0} \tau_{1}}{\pi} \ln 2+c_{0}=-\frac{a_{0} \tau_{1}}{\pi} \ln \rho+a_{0} \tau_{1} g^{+}\left(0, \frac{\pi}{d_{+}}\right)-\frac{a_{0} \tau_{2}}{\pi}, \\
\frac{a_{0} \tau_{1}}{\pi} \ln \rho+\frac{a_{0} \tau_{1}}{\pi} \ln 2+c_{0}=\frac{a_{0} \tau_{1}}{\pi} \ln \rho-a_{0} \tau_{1} g^{-}\left(0, \frac{\pi}{d_{+}}\right)+\frac{a_{0} \tau_{2}}{\pi} .
\end{array}\right.
$$

Система (13) дает значения $c_{0}$ и $\tau_{2}$ : 


$$
\begin{aligned}
& c_{0}=\frac{a_{0} \pi}{4 d_{+}}\left(g^{+}\left(0, \frac{\pi}{d_{+}}\right)-g^{-}\left(0, \frac{\pi}{d_{+}}\right)\right) \\
& \tau_{2}=\frac{\pi^{2}}{4 d_{+}}\left(g^{+}\left(0, \frac{\pi}{d_{+}}\right)+g^{-}\left(0, \frac{\pi}{d_{+}}\right)\right)+\frac{\pi}{2 d_{+}} \ln 2 .
\end{aligned}
$$

Особенно интересно найти мнимую часть квазисобственного значения, которая определяет время жизни соответствующего резонансного состояния. Принимая во внимание, что

$$
\operatorname{Im} g^{+}\left(x_{i}, \frac{\pi}{d_{+}}\right)=-\frac{1}{2 \pi}, \quad \operatorname{Im} g^{-}\left(x_{i}, \frac{\pi}{d_{+}}\right)=-\frac{d_{+}}{2 \pi d_{-}}
$$

получаем

$$
\operatorname{Im} \tau_{2}=-\frac{\pi}{8}\left(\frac{1}{d_{+}}+\frac{1}{d_{-}}\right)
$$

Теперь рассмотрим случай двух одинаковых волноводов $\left(d_{+}=d_{-}=d\right)$. В этом случае $G^{+}\left(x, 0, k_{a}\right)=G^{-}\left(x, 0, k_{a}\right)$ и, следовательно, соотношения (7) и (8) заменяются на

$G^{ \pm}\left(x, 0, k_{a}\right)=\frac{1}{d \sqrt{\pi^{2} d^{-2}-k_{a}^{2}}} \cos \frac{\pi x_{2}}{d} e^{-\sqrt{\pi^{2} d^{-2}-k_{a}^{2}}}\left|x_{1}\right|-\frac{1}{\pi} \ln \left(\frac{a}{d_{-}}\right)-\frac{1}{\pi} \ln \rho+g\left(x, \frac{\pi}{d}\right)$.

Схема аналогична той, что использовалась в случае различных волноводов. Согласование членов порядка $a^{0}$ дает следующие уравнения вместо (10):

$$
a_{0}\left(\frac{1}{d}-\frac{\tau_{1}}{\pi}\right)=v_{0}(\xi)=-a_{0}\left(\frac{1}{d}-\frac{\tau_{1}}{\pi}\right) .
$$

Значит, мы получаем $v_{0}(\xi)=0$, и

$$
\tau_{1}=\frac{\pi}{d}
$$

Аналогично получаем для членов порядка $\ln ^{-1}\left(a / d_{-}\right)($ср. с $(13))$

$$
\left\{\begin{array}{l}
-\frac{a_{0} \tau_{1}}{\pi} \ln \rho-\frac{a_{0} \tau_{1}}{\pi} \ln 2+c_{0}=-\frac{a_{0} \tau_{1}}{\pi} \ln \rho+a_{0} \tau_{1} g\left(0, \frac{\pi}{d}\right)-\frac{a_{0} \tau_{2}}{\pi} \\
\frac{a_{0} \tau_{1}}{\pi} \ln \rho+\frac{a_{0} \tau_{1}}{\pi} \ln 2+c_{0}=\frac{a_{0} \tau_{1}}{\pi} \ln \rho-a_{0} \tau_{1} g\left(0, \frac{\pi}{d}\right)+\frac{a_{0} \tau_{2}}{\pi} .
\end{array}\right.
$$

Решая систему, получаем $c_{0}=0$ и

$$
\tau_{2}=\frac{\pi^{2}}{d} g\left(0, \frac{\pi}{d}\right)+\frac{\pi}{d} \ln 2 .
$$

Мнимая часть $\tau_{2}$ в этом случае такова:

$$
\operatorname{Im} \tau_{2}=-\frac{\pi}{2 d}
$$

Объединяя результаты, получаем следующую теорему. 
ТЕОрема 2. Первые члены асимптотического разложения квазисобственного значения $k_{a}^{2}$, близкого $к$ границе ветви непрерьвного спектра $\pi^{2} / d_{+}^{2}$ оператора Лапласа с краевым условием Неймана для двух полос, связанных через малое отверстие ииринъ $2 a$, имеет вид

$$
\sqrt{\frac{\pi^{2}}{d_{+}^{2}}-k_{a}^{2}}=\tau_{1} \ln ^{-1}\left(\frac{a}{d_{-}}\right)+\tau_{2} \ln ^{-2}\left(\frac{a}{d_{-}}\right)+\cdots,
$$

гde

$$
\begin{gathered}
\tau_{1}= \begin{cases}\frac{\pi}{2 d_{+}}, & d_{+}>d_{-}, \\
\frac{\pi}{d}, & d_{+}=d_{-}=d,\end{cases} \\
\tau_{2}= \begin{cases}\frac{\pi^{2}}{4 d_{+}}\left(g^{+}\left(0, \frac{\pi}{d_{+}}\right)+g^{-}\left(0, \frac{\pi}{d_{+}}\right)\right)+\frac{\pi}{2 d_{+}} \ln 2, & d_{+}>d_{-}, \\
\frac{\pi^{2}}{d} g\left(0, \frac{\pi}{d}\right)+\frac{\pi}{d} \ln 2, & d_{+}=d_{-}=d,\end{cases} \\
\operatorname{Im} \tau_{2}= \begin{cases}-\frac{\pi}{8}\left(\frac{1}{d_{+}}+\frac{1}{d_{-}}\right), & d_{+}>d_{-}, \\
-\frac{\pi}{2 d}, & d_{+}=d_{-}=d .\end{cases}
\end{gathered}
$$

ЗАмечАниЕ 2. Оценка остаточного члена (т.е. обоснование асимптотического разложения) будет получена в последнем разделе (см. (39)).

\section{4. РАССЕЯНИЕ}

Интересно проанализировать задачу рассеяния плоской волны $e^{i k x}$. Рассмотрение задачи рассеяния в рамках асимптотического подхода имеет специфические черты. Если волновое число падающей волны фиксировано и отличается от резонансного значения, то характеристический параметр $k_{a}$ мал, и рассеяния волны отверстием при достаточно малом $a$ нет. Вот почему соответствующие члены асимптотик будут нулевыми. Чтобы преодолеть это препятствие, мы предположим, что $k$ близок к резонансу, и отклонение от резонанса характеризуется отличием $c$ от $\tau_{2}$ :

$$
\sqrt{\frac{\pi^{2}}{d_{+}^{2}}-k^{2}}=\tau_{1} \ln ^{-1}\left(\frac{a}{d_{-}}\right)+c \ln ^{-2}\left(\frac{a}{d_{-}}\right)+\cdots .
$$

Мы используем метод согласования (см. выше). Значение $\tau_{1}$ дается формулой (11).

Получаем

$$
\frac{1}{d_{+} \sqrt{\pi^{2} d_{+}^{-2}-k^{2}}}=\frac{1}{d_{+} \tau_{1}} \ln \left(\frac{a}{d_{-}}\right)-\frac{c}{d_{+} \tau_{1}^{2}}+\cdots .
$$

Асимптотика решения $\psi(x)$ задачи рассеяния падающей волны $e^{i k x}$, которая распространяется в $\Omega^{+}$, имеет вид

$$
\psi(x)= \begin{cases}e^{-i k x}+\alpha_{1} G^{+}(x, 0, k)+\cdots, & x \in \Omega^{+} \backslash S_{\sqrt{a}}, \\ v_{-1}\left(\frac{x}{a}\right) \ln \left(\frac{a}{d_{-}}\right)+v_{0}\left(\frac{x}{a}\right)+v_{1}\left(\frac{x}{a}\right) \ln ^{-1}\left(\frac{a}{d_{-}}\right)+\cdots, & x \in S_{2 \sqrt{a}} \\ -\alpha_{1} G^{-}(x, 0, k)+\cdots, & x \in \Omega^{-} \backslash S_{\sqrt{a}} .\end{cases}
$$


Функция Грина при $x \rightarrow \infty$ содержит член $e^{i k x}$ :

$$
\psi^{+}(x)=e^{-i k x}+\alpha_{1}\left(\frac{1}{2 i k d_{+}} e^{-i k x}+\cdots\right) .
$$

Коэффициент прохождения $T$ имеет вид

$$
T=\left|1-\frac{i \alpha_{1}}{2 \pi}\right|^{2}
$$

После замены переменных $\xi=x / a, \rho=|\xi|$ получаем асимптотику функции Грина:

$$
\begin{gathered}
G^{+}(\xi, 0, k)=\frac{1}{d_{+} \tau_{1}} \ln \left(\frac{a}{d_{-}}\right)-\frac{c}{d_{+} \tau_{1}^{2}}-\frac{1}{\pi} \ln \left(\frac{a}{d_{-}}\right)-\frac{1}{\pi} \ln \rho+g^{+}\left(\xi, \frac{\pi}{d_{+}}\right)+\cdots, \\
G^{-}(\xi, 0, k)=-\frac{1}{\pi} \ln \left(\frac{a}{d_{-}}\right)-\frac{1}{\pi} \ln \rho+g^{-}\left(\xi, \frac{\pi}{d_{+}}\right) .
\end{gathered}
$$

Первые члены асимптотики волновой функции таковы:

$$
\begin{aligned}
& \psi^{+}(x)=1+\alpha_{1}\left[-\frac{c}{d_{+} \tau_{1}^{2}}+\left(-\frac{1}{\pi}+\frac{1}{d_{+} \tau_{1}}\right) \ln \left(\frac{a}{d_{-}}\right)+g^{+}\left(0, \frac{\pi}{d_{+}}\right)-\frac{1}{\pi} \ln \rho\right]+o(1), \\
& \psi_{a}^{-}(x)=-\alpha_{1}\left[-\frac{1}{\pi} \ln \rho+g^{-}\left(0, \frac{\pi}{d_{+}}\right)-\frac{1}{\pi} \ln \left(\frac{a}{d_{-}}\right)\right]+o(1) .
\end{aligned}
$$

Чтобы найти $\alpha_{1}$, рассмотрим члены порядка $a^{0}$. Чтобы произвести согласование, выбираем в качестве функции $v_{0}(\xi)$

$$
\begin{gathered}
v_{0}(\xi)= \begin{cases}-\frac{\alpha_{1}}{\pi} X_{0}(\xi)+A, & \xi_{2}>0 \\
\frac{\alpha_{1}}{\pi} X_{0}(\xi)+A, & \xi_{2}<0\end{cases} \\
X_{0}(\zeta)=\ln \left(\zeta+\sqrt{\zeta^{2}-1}\right)
\end{gathered}
$$

где $A$ - некоторая постоянная. Процедура согласования приводит к определению $\alpha_{1}$ :

$$
\alpha_{1}(c)=\left[-\frac{2}{\pi} \ln 2+\frac{4 c d_{+}}{\pi^{2}}-\left(g^{+}\left(0, \frac{\pi}{d_{+}}\right)+g^{-}\left(0, \frac{\pi}{d_{+}}\right)\right)\right]^{-1} .
$$

Рассмотрим случай двух одинаковых волноводов $\left(d_{+}=d_{-}=d\right)$. Используя аналогичную процедуру, находим

$$
\alpha_{1}(c)=\frac{1}{2}\left(\frac{c d}{\pi^{2}}-\frac{1}{\pi} \ln 2-g\left(0, \frac{\pi}{d}\right)\right)^{-1} .
$$

ТЕОРема 3. Коэффиииент прохождения плоской волны в двух двумерных волноводах, связанных через малое отверстие, имеет вид

$$
T=\left|1-\frac{i \alpha_{1}}{2 \pi}\right|^{2}
$$


где

$$
\alpha_{1}= \begin{cases}{\left[\frac{4 c d_{+}}{\pi^{2}}-\frac{2}{\pi} \ln 2-\left(g^{+}\left(0, \frac{\pi}{d_{+}}\right)+g^{-}\left(0, \frac{\pi}{d_{+}}\right)\right)\right]^{-1},} & d_{+}>d_{-}, \\ \left(\frac{2 c d}{\pi^{2}}-\frac{2}{\pi} \ln 2-g\left(0, \frac{\pi}{d}\right)\right)^{-1}, & d_{+}=d_{-}=d,\end{cases}
$$

и с берется из формуль (19).

Мы представляем в статье асимптотическое разложение для квазисобственного значения, близкого к границе $\pi^{2} / d_{+}^{2}$. Можно видеть, что наше рассмотрение подходит и для границ других ветвей непрерывного спектра. Интересно сравнить результат с соответствующими асимптотиками для оператора Лапласа с граничным условием Дирихле, которые получены в работе [6]. А именно, следующее асимптотическое разложение имеет место для квазисобственного значения, близкого к границе $\pi^{2} / d_{-}^{2}$ :

$$
\left(\frac{\pi^{2}}{d_{-}^{2}}-k_{a}^{2}\right)^{1 / 2}=k_{20} a^{2}+k_{40} a^{4}+k_{41} a^{4} \ln \frac{a}{a_{0}}+o\left(a^{4}\right)
$$

где

$$
\begin{array}{rlrl}
k_{0}^{2} & =\frac{\pi^{2}}{d_{-}^{2}}, & k_{20} & =\frac{\pi^{3}}{4 d_{-}^{3}}, \\
k_{40}=\frac{\pi^{5}}{16 d_{-}^{2} d_{+}^{2} \sqrt{d_{+}^{2}-d_{-}^{2}}}, & k_{41}=\frac{\pi^{4}}{16 d_{-}^{2}}\left(\frac{3 \pi}{8 d_{-}^{3}}-\frac{1}{d_{-}}\left(g_{x}^{+}+g_{x}^{-}\right)\right) .
\end{array}
$$

Здесь $g_{x}^{ \pm}-$значение в точке $(0,0)$ нормальных производных регулярных частей функций Грина соответствующих волноводов (без отверстий). Сравнение разложения (30) и теоремы 2 показывает, что асимптотики для случаев Дирихле и Неймана имеют различный порядок относительно $a$. А именно, влияние отверстия больше для случая Неймана. Это естественно, поскольку невозмущенное решение (без отверстия) имеет максимум модуля на границе в случае Неймана и узел (нуль) в случае Дирихле.

Тот факт, что положение резонанса различно в случаях Дирихле и Неймана, может быть использован при конструировании наноустройств. В структурах с перемежающимися магнитными и немагнитными слоями (см. введение) возможно, что волновая функция электрона с одной ориентацией спина удовлетворяет краевому условию Дирихле, в то время как волновая функция электрона с другой ориентацией спина подчиняется условию Неймана. Это означает, что при подходящем выборе параметров имеется резонанс для электрона с одной ориентацией спина (т.е. коэффициент прохождения существенно меньше 1 , а коэффициент отражения ненулевой) и отсутствие резонанса для электрона с другой ориентацией спина (т.е. коэффициент отражения равен нулю). Следовательно, получаем поток поляризованных по спину отраженных электронов. Что касается прошедшего электронного пучка, он будет частично поляризован. Если мы хотим получить полностью поляризованный прошедший пучок, то можно использовать систему с большим числом отверстий связи, которые достаточно удалены друг от друга (чтобы избежать интерференционных эффектов); тогда поляризационный эффект накапливается. 
Форма асимптотического ряда определяется типом граничного условия на линии, в которой имеется отверстие. Вот почему случай комбинированных условий Дирихле-Неймана (с условием Неймана на разделяющей линии) абсолютно аналогичен описанному выше. Единственное изменение в формулах связано с изменением собственных функций в поперечном сечении для невозмущенного случая (в формуле для невозмущенной функции Грина).

Можно видеть, что наша схема анализа позволяет заменить лапласиан оператором с гладким потенциалом $-\Delta+u\left(x_{2}\right)$. Это возможно, поскольку для получения асимптотической формулы для резонанса достаточно двух членов асимптотического разложения, а для первых членов имеем уравнение Лапласа (см. (9) с учетом следующей замены переменных: $\xi=x / a)$. Необходимо лишь заменить собственную функцию для поперечного сечения $\left(\cos \left(\pi n x_{2} / d\right)\right)$ для оператора $-d^{2} / d x_{2}^{2}$ соответствующей функцией для оператора Шредингера $-d^{2} / d x_{2}^{2}+u\left(x_{2}\right)$. Члены $g^{ \pm}$в этом случае также другие.

\section{5. ОЦЕНКА НЕВЯЗКИ И АНАЛИЗ АСИМПТОТИК}

Используя ранее полученные оценки $\hat{\psi}_{a}\left(x, \hat{k}_{a}\right)$ квазисобственной функции $\psi_{a}\left(x, k_{a}\right)$ в трех областях $\left(\Omega^{ \pm} \backslash S_{2 \sqrt{a}}, S_{\sqrt{a}}, S_{2 \sqrt{a}} \backslash S_{\sqrt{a}}\right)$, можно получить оценку невязки:

$$
\left\|\left(\Delta+\hat{k}_{a}^{2}\right) \hat{\psi}_{a}\left(x, \hat{k}_{a}\right)\right\|_{\mathbf{L}^{2}} \leqslant C a^{1 / 2} .
$$

Чтобы обосновать найденную выше формальную асимптотику, используем подход, аналогичный предложенному в работе [12]. Существование квазисобственного значения $k_{a}^{2}$ (с соответствующей квазисобственной функцией $\psi_{a}$ ) вблизи границы ветви непрерывного спектра было доказано выше. Используем представления $\psi_{a}=\hat{\psi}_{a}+\varphi_{a}, k_{a}^{2}=\hat{k}_{a}^{2}+m_{a}^{2}$. Значит, уравнение для $\varphi_{a}, m_{a}^{2}$ (остаточный член) имеет вид:

$$
\left(\Delta+\hat{k}_{a}^{2}+m_{a}^{2}\right) \varphi_{a}+m_{a}^{2} \hat{\psi}_{a}=-\Delta \hat{\psi}_{a}-\hat{k}_{a}^{2} \hat{\psi}_{a} .
$$

Правая часть уравнения (31) была оценена в начале раздела. Нам бы хотелось получить оценку для $m_{a}^{2}$. Можно видеть, что вследствие (6), (8) имеем $m_{a}^{2} \hat{\psi}_{a}+$ $\Delta \hat{\psi}_{a}+\hat{k}_{a}^{2} \hat{\psi}_{a}=\ln ^{-1} N_{a} \varphi_{a}$. Здесь $N_{a}$ - линейный ограниченный оператор. Решение уравнения

$$
\left(\Delta+\hat{k}_{a}^{2}+m_{a}^{2}\right) \varphi_{a}=f_{a}
$$

представимо в форме

$$
\varphi_{a}=A\left(m_{a}\right) f_{a},
$$

где $A\left(m_{a}\right)$ - мероморфная функция $m_{a}$. Значит, уравнение $(31)$ преобразуется к виду

$$
f_{a}+\ln ^{-1} a N_{a} A\left(m_{a}\right) f_{a}=0 .
$$

Следуя предложенной в работе [12] схеме, представим $A\left(m_{a}\right) f_{a}$ следующим образом:

$$
A\left(m_{a}\right) f_{a}=\frac{K_{0}\left(f_{a}\right) \hat{\psi}_{a}}{m_{a}}+A_{1}\left(m_{a}\right) f_{a},
$$


где $A_{1}\left(m_{a}\right)$ голоморфна по $m_{a}$. Это представление получено тем же путем, что и соответствующее в работе [12]. А именно, мы обрезаем область полосой $\left|x_{1}\right| \leqslant$ $R$, умножаем уравнение $(32)$ на $\hat{\psi}_{a}$ и интегрируем по области. После выполнения несложных операций заключаем, что

$$
K_{0}\left(f_{a}\right)=\int_{\Omega^{ \pm}} f_{a} \hat{\psi}_{a} d^{2} x
$$

Это и дает выражение (34).

Подставляя (34) в (33), получаем

$$
f_{a}+\ln ^{-1} a \frac{K_{0}\left(f_{a}\right) N_{a} \hat{\psi}_{a}}{m_{a}}+\ln ^{-1} a N_{a} A_{1}\left(m_{a}\right) f_{a}=0 .
$$

Оператор $N_{a} A_{1}\left(m_{a}\right)$ непрерывен, значит, для достаточно малых а обратный оператор $B\left(m_{a}, a\right)=\left(I+\ln ^{-1}\left(a / d_{-}\right) N_{a} A_{1}\left(m_{a}\right)\right)^{-1}$ существует и сходится к единичному при $a \rightarrow 0$ равномерно по $m_{a}$. Применяя $B\left(m_{a}, a\right)$ к уравнению $(36)$, получаем

$$
f_{a}+\ln ^{-1}\left(\frac{a}{d_{-}}\right) \frac{1}{m_{a}} K_{0}\left(f_{a}\right) B\left(m_{a}, a\right) N_{a} \hat{\psi}_{a}=0 .
$$

Заметим, что $K_{0}\left(f_{a}\right)$ не равно нулю (в противном случае $f_{a}$ было бы нулем). Следовательно, применяя оператор $K_{0}$ к $(37)$ и деля полученное уравнение на $K_{0}\left(f_{a}\right)$ приходим к уравнению для $m_{a}$ :

$$
m_{a}=-\ln ^{-1}\left(\frac{a}{d_{-}}\right) K_{0}\left(B\left(m_{a}, a\right) N_{a} \hat{\psi}_{a}\right) .
$$

Принимая во внимание, что $K_{0}\left(B(m, a) N_{a} \hat{\psi}_{a}\right)$ голоморфна по $m$ и непрерывна как функция $(m, a)$, получаем для достаточно малых $a$ следующую оценку на границе малой окрестности нуля:

$$
|m|>\left|\ln ^{-1}\left(\frac{a}{d_{-}}\right) K_{0}\left(B\left(m_{a}, a\right) N_{a} \hat{\psi}_{a}\right)\right| .
$$

По теореме Руше (для операторнозначных функций [16]) функции $m$ и $m+$ $\ln ^{-1}\left(a / d_{-}\right) K_{0}\left(B\left(m_{a}, a\right) N_{a} \hat{\psi}_{a}\right)$ имеют одинаковое число нулей в этой области. Следовательно, уравнение (38) имеет единственное решение в малой окрестности нуля для достаточно малых $a$. Учитывая оценки для $\hat{\psi}_{a}$ и $f_{a}$ (оценка невязки, см. предыдущий раздел), свойства $B\left(m_{a}, a\right)$ и $(35)$, приходим к следующей оценке остаточного члена $m_{a}$ в асимптотике (см. теорему 2): $m_{a}=o\left(\ln ^{-2}\left(a / d_{-}\right)\right)$. В результате получаем обоснование асимптотики в теореме 2 :

$$
\sqrt{\frac{\pi^{2}}{d_{+}^{2}}-k_{a}^{2}}=\tau_{1} \ln ^{-1}\left(\frac{a}{d_{-}}\right)+\tau_{2} \ln ^{-2}\left(\frac{a}{d_{-}}\right)+o\left(\ln ^{-2}\left(\frac{a}{d_{-}}\right)\right) .
$$

Заметим, что предложенный подход можно использовать для получения следующих членов асимптотического разложения и для его обоснования. 
Благодарности. Работа поддержана РФФИ (грант № 05-03-32576) и программой "Интеграция науки и высшего образования" (государственный контракт № П0045/2307). Авторы благодарят В. М. Уздина за плодотворные обсуждения.

\section{Список литературы}

[1] P. Exner, S. Vugalter. Ann. Inst. H. Poincaré. 1996. V. 65. № 1. P. 109-123.

[2] P. Exner, S. Vugalter. J. Phys. A. 1997. V. 30. P. 7863-7878.

[3] I. Yu. Popov. Rep. Math. Phys. 1999. V. 43. № 3. P. 427-437; J. Math. Phys. 2002. V. 43. № 1. P. 215-234.

[4] И. Ю. Попов. ТМФ. 2002. Т. 131. № 3. С. 407-418.

[5] J. Dittrich, J. Kriz. J. Math. Phys. 2002. V. 43. P. 3892-3915.

[6] S. V. Frolov, I. Yu. Popov. J. Math. Phys. 2000. V. 41. № 7. P. 4391-4405.

[7] S. V. Frolov, I. Yu. Popov. J. Phys. A. 2003. V. 36. P. 1655-1670.

[8] Ch. Kunze. Phys. Rev. B. 1993. V. 48. P. 14338-14346.

[9] P. Bruno. Phys. Rev. B. 1995. V. 52. № 1. P. 411-439; C.D. Potter, R. Shad, P. Belien, G. Verbanck, V.V. Moshalkov, Y. Bruynseraede, N. Schafer, R. Schafer, P. Grunberg. Phys. Rev. B. 1994. V. 49. № 22. P. 16055-16057; P. Grunberg. J. Phys.: Condens. Matter. 2001. V. 13. P. 7691-7706.

[10] V.M. Uzdin, N.S. Yartseva. J. Magn. Magn. Mater. 1996. V. 156. P. 193-194.

[11] А. А. Арсенъев. Матем. сб. 2003. Т. 194. № 1. С. 3-22.

[12] P. Borisov, P. Exner, R. Gadilshin. J. Math. Phys. 2002. V. 43. № 12. P. 6265-6278.

[13] Р. Р. Гадыльшин. УМН. 1997. Т. 52. № 1. С. 3-76.

[14] А. М. Ильин. Согласование асимптотических разложений решений краевых задач. М.: Наука, 1989.

[15] Н. С. Ландкоф. Основы современной теории потенциала. М.: Наука, 1966.

[16] И. Ц. Гохберг, Е.И. Сигал. Матем. сб. 1971. Т. 84. № 4. С. 607-629.

Поступила в редакцию 2.II.2005 г., после доработки 16.IX.2005 г. 